\title{
Detection of Atrial Fibrillation From Short ECGs: Minimalistic Complexity Analysis for Feature-Based Classifiers
}

\author{
Anara Abdukalikova ${ }^{1}$, Denis Kleyko $^{1}$, Evgeny Osipov $^{1}$, Urban Wiklund ${ }^{2}$ \\ ${ }^{1}$ Luleå University of Technology, Luleå, Sweden \\ ${ }^{2}$ Umeå University, Umeå, Sweden
}

\begin{abstract}
In order to facilitate data-driven solutions for early detection of atrial fibrillation (AF), the 2017 CinC conference challenge was devoted to automatic AF classification based on short ECG recordings. The proposed solutions concentrated on maximizing the classifiers $F_{1}$ score, whereas the complexity of the classifiers was not considered. However, we argue that this must be addressed as complexity places restrictions on the applicability of inexpensive devices for AF monitoring outside hospitals. Therefore, this study investigates the feasibility of complexity reduction by analyzing one of the solutions presented for the challenge.
\end{abstract}

\section{Introduction}

This paper is related to the studying of detection of one of the cardiovascular diseases - Atrial Fibrillation (AF) arrhythmia. AF is a supraventricular tachyarrhythmia which is represented by inconstant atrial activation and, therefore, dysregulation of atrial contractions. This type of arrhythmia has a severe influence on the heart health condition and can cause congestive heart failure, stroke and even lead to death. The work on solving the problem of early AF detection has been done before and different detection techniques were used. Although most of the previous studies showed quite high and promising results, they had a number of limitations. Small sized datasets were presented by carefully selected signals of only two classes: $\mathrm{AF}$ and normal. In this paper we use the database of signals from the 2017 PhysioNet/CinC Challenge and investigate one of the solutions presented for the challenge. However, the original 2017 PhysioNet/CinC Challenge formulation did not account for the future deployment on the resource-limited devices for patients to use on a daily basis. This requirement limits the computational complexity of a solution. Therefore, we explore a solution for reducing necessary computations by investigating feature selection method called Reduced Feature Elimination (RFE).

\section{2017 PhysioNet/CinC Challenge}

The limitations mentioned for the previous studies of AF detection were aimed to be solved in the 2017 PhysioNet/CinC Challenge. The purpose of this challenge was to develop an accurate mechanism for AF detection among four different types of signals: AF, normal, other (presented by some other cardiac abnormalities) and noise. The dataset included 8528 single lead ECG recordings for training and 3658 ECG signals for testing, which were not disclosed to the public [1]. The distribution between different classes was as follows: Normal 5076 recordings; AF 758 recordings; Other 2415 recordings; Noise 279 recordings. All recordings had a duration varying from $9 \mathrm{~s}$ to approximately $61 \mathrm{~s}$ and were sampled at $300 \mathrm{~Hz}$. In comparison to previous studies, the challenge of this competition was that the dataset comprised bigger amount of signals and the detection had to be realized among four different classes with unequal number of samples in each. Additionally, each of the signals had a short duration, which also increases the complexity for AF detection mechanism, since usually ECG signals are recorded with 12 leads for a longer duration. Thus, the detection mechanism has to be able to properly extract meaningful features to accurately detect abnormalities in signal. Nevertheless, the evaluation of the best performing solutions [1] showed that it was possible to achieve the $F_{1}$ score of 0.8926 and 0.83 on the training and testing sets respectively.

\section{Motivation}

Since AF is considered to be the most common form of heart arrhythmia and significantly influence patients health, its early recognition is crucial and requires reliable tools for its detection. Practical limitations require that the diagnosis can be done outside a hospital using inexpensive equipment and without an involvement of medical staff. Also, the time required to make a test should be short. As it was mentioned earlier, the deployment of AF detection mechanism should be realized on the resourcelimited devices. Local processing is preferable because 
of intermittent connectivity to a server and data privacy concerns. However, this demands simple computations including preprocessing, feature extraction, running a classifier, etc. Thus, the practical limitation is the computational simplicity of the solution and small memory footprint.

\section{Methods}

\subsection{Features}

This paper uses the solution to the 2017 CinC challenge presented in [2] by F. Andreotti et al. The solution in [2] extracts 171 different features from filtered segmented ECG recordings where the number of segments depends on the length of the recording. Therefore, for each recording the mean values across all segments were used for further processing. The features were extracted using HRV metrics, signal quality indices, and also morphological ones. In addition to time domain, frequency domain, and non-linear HRV metrics, metrics based on clustering of beats on Poincare plots were used. Extracting this large number of features in the real-time predicting systems deployed on resource-limited devices is highly doubtable due to computational and time constraints. Therefore, this paper explores ways of reducing the number of features by using the RFE method as well as a statistical approach.

\subsection{Selection of features}

Two approaches to feature selection briefly described below were used for reducing the number of features so that it would take less computations and time to extract them for new recordings.

\subsubsection{Recursive Feature Elimination}

The RFE method [3] is a greedy optimization technique used to find the subset of best performing features. It repeatedly builds classification models, keeps the worst or the best feature subsets aside and afterwards computes the accuracy. This process is repeated until all features are exhausted. Next, RFE evaluates and ranks all the features based on the order of their elimination. Finally, it provides the indices of the best performing features, which form the subset of the pre-specified size. It is worth noting that the features forming the best performing subset by means of their ranking are not necessarily individually the most important. These features perform well only in combination with the other features in the corresponding subset. During the experiments, the RFE function from Python scikitlearn machine learning library was used to implement the technique.

\subsubsection{Statistical approach}

The statistical approach included calculating $p$-values for each feature using Kruskal-Wallis and multiple comparison tests. The $p$-values were calculated as the sum of all scores after the multiple comparison test. It allows selecting features where all classes differ. Then the desired number of features could be chosen among the features with the highest $p$-values. During the experiments, kruskalwallis and multcompare methods in Matlab were used to implement the statistical approach.

\subsection{Classification method}

For the sake of brevity, this paper reports only the performance obtained for one classification method. The chosen method is Random Forest Classifier since it was reported to demonstrate the highest classification performance for the solution in [2].

\section{Results}

Table 1. Classification performance of Random Forest Classifier for different number of features selected by the RFE method.

\begin{tabular}{|c|c|c|}
\hline Number of features & Accuracy & $F_{1}$ score \\
\hline 5 & 0,78 & 0,70 \\
\hline 8 (only time domain) & 0,78 & 0,65 \\
\hline 10 & 0,81 & 0,74 \\
\hline 15 & 0,82 & 0,75 \\
\hline 20 & 0,83 & 0,75 \\
\hline 171 (the whole set) & 0.83 & 0.75 \\
\hline
\end{tabular}

This section presents the classification performance obtained for the reduced number of features when using the RFE method and the statistical approach. First, to obtain the reference performance Random Forest Classifier was trained on the whole feature set. Next, we calculated the performance of Random Forest Classifier on the best feature subsets chosen by the RFE method for several subset sizes. The number of selected features varied between 5 and 20. The performance was measured with accuracy and mean $F_{1}$ score using 5-fold cross-validation. The results reported in Table 1 were obtained by averaging ten independent runs of the classifier.

The accuracy and mean $F_{1}$ score on the whole set of 171 features were 0,83 and 0,75 respectively. The results showed that in comparison to the usage of all 171 features using the subset of only 5 best features worsened accuracy by $6.0 \%$ and $F_{1}$ score by $6.7 \%$. On the other hand, the difference to the reference classifier when using 10 features was only $2.4 \%$ and $1.3 \%$ respectively. There was no significant performance degradation for 15 and 20 features. The 
subset of 15 features was chosen as the resulting one for future feature extraction from new signals and for training other classifiers. This subset was more appealing to use, since its feature extraction did not require any frequency domain computations. It included features extracted from Recurrence Quantification Analysis (RQA), Poincare plot, Signal Quality Indices (SQI) metrics, 3 morphological and 2 residual features. Using only 8 features extracted from the temporal domain was comparable to 5 best features in terms of accuracy but was $7.1 \%$ worse in terms of $F_{1}$ score.

Table 2 presents features included in the subsets formed by the RFE method. The table includes only three subsets, since there was no big difference in the results obtained for 15 and 20 features subsets.

Table 2. Top ranked features for best feature subsets.

\begin{tabular}{|c|c|c|c|c|}
\hline \# & $\begin{array}{c}\text { Name of a } \\
\text { feature }\end{array}$ & $\begin{array}{c}5 \\
\text { features } \\
\text { subset }\end{array}$ & $\begin{array}{c}10 \\
\text { features } \\
\text { subset }\end{array}$ & $\begin{array}{c}15 \\
\text { features } \\
\text { subset }\end{array}$ \\
\hline 1 & SampleAFEv & $\checkmark$ & $\checkmark$ & $\checkmark$ \\
\hline 2 & RR & $x$ & $\sqrt{ }$ & $\checkmark$ \\
\hline 3 & TKEO1 & $x$ & $\sqrt{ }$ & $\sqrt{ }$ \\
\hline 4 & medRR & $x$ & $x$ & $\checkmark$ \\
\hline 5 & iqrdRR & $x$ & $x$ & $\checkmark$ \\
\hline 6 & DistNext & $x$ & $\checkmark$ & $\sqrt{ }$ \\
\hline 7 & ClustDistSTD & $x$ & $x$ & $\sqrt{ }$ \\
\hline 8 & $\operatorname{rad} 1$ & $x$ & $\checkmark$ & $x$ \\
\hline 9 & $\operatorname{rad} 2$ & $\checkmark$ & $\sqrt{ }$ & $\sqrt{ }$ \\
\hline 10 & DistNextnS & $\checkmark$ & $x$ & $x$ \\
\hline 11 & rsqi3 & $\sqrt{ }$ & $\checkmark$ & $x$ \\
\hline 12 & rsqi5 & $x$ & $x$ & $\sqrt{ }$ \\
\hline 13 & csqi2 & $x$ & $x$ & $\sqrt{ }$ \\
\hline 14 & csqi5 & $x$ & $\sqrt{ }$ & $x$ \\
\hline 15 & res1 & $\checkmark$ & $\checkmark$ & $\sqrt{ }$ \\
\hline 16 & res2 & $x$ & $x$ & $\checkmark$ \\
\hline 17 & Pheight & $x$ & $\checkmark$ & $x$ \\
\hline 18 & QRSpow & $x$ & $x$ & $\checkmark$ \\
\hline 19 & PheigtNorm & $x$ & $x$ & $\sqrt{ }$ \\
\hline 20 & RRlen & $x$ & $x$ & $\checkmark$ \\
\hline
\end{tabular}

Figure 1 presents distribution of 15 features selected by the RFE for each class. Feature numbers correspond to the order in Table 1. In general, not all the features demonstrate distinct separation between the classes (e.g., \#2), however, it is clear that there are features (e.g., \#1, \#6) where classes have different values.

Table 3 presents the confusion matrix obtained on 5-fold cross-validation for a single run of the Random Forest classifier trained on all 171 features. The cross-validation accuracy on the data was 0.83 while mean $F_{1}$ score was 0.74 .

Table 4 presents the confusion matrix obtained on 5 -fold cross-validation for a single run of the Random Forest classifier trained on the best 15 features selected by RFE. The
Table 3. Confusion matrix for all features.

\begin{tabular}{|c|c|c|c|c|c|}
\hline & \multicolumn{4}{|c|}{ Predicted } \\
\hline & & $\mathrm{AF}$ & Normal & Other & Noise \\
\hline \multirow{4}{*}{ 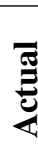 } & $\mathrm{AF}$ & 575 & 36 & 135 & 12 \\
\hline & Normal & 16 & 4698 & 339 & 23 \\
\hline & Other & 83 & 647 & 1655 & 30 \\
\hline & Noise & 10 & 72 & 66 & 131 \\
\hline
\end{tabular}

cross-validation accuracy on the data was 0.83 while mean $F_{1}$ score was 0.74 .

Table 4. Confusion matrix for 15 features selected by the RFE method.

\begin{tabular}{|c|c|c|c|c|c|}
\hline & \multicolumn{4}{|c|}{ Predicted } \\
\hline & & $\mathrm{AF}$ & Normal & Other & Noise \\
\hline \multirow{4}{*}{ } & $\mathrm{AF}$ & 572 & 32 & 143 & 10 \\
\hline & Normal & 23 & 4692 & 341 & 20 \\
\hline & Other & 102 & 640 & 1638 & 35 \\
\hline & Noise & 14 & 71 & 51 & 143 \\
\hline
\end{tabular}

Table 5 presents the confusion matrix obtained on 5-fold cross-validation for a single run of the Random Forest classifier trained on 15 features selected by the statistical approach. The cross-validation accuracy on the data was 0.78 while mean $F_{1}$ score was 0.69 .

Table 5. Confusion matrix for 15 selected features by the statistical approach.

\begin{tabular}{|c|c|c|c|c|c|}
\hline & \multicolumn{4}{|c|}{ Predicted } \\
\hline & & $\mathrm{AF}$ & Normal & Other & Noise \\
\hline \multirow{4}{*}{ 胥 } & $\mathrm{AF}$ & 572 & 38 & 133 & 15 \\
\hline & Normal & 25 & 4604 & 418 & 29 \\
\hline & Other & 112 & 893 & 1369 & 41 \\
\hline & Noise & 16 & 84 & 52 & 127 \\
\hline
\end{tabular}

The results in Tables 3-5 demonstrate that the classification performance of the RFE-formed subset corresponds to that of the full set, while the classification performance of the subset obtained with the statistical approach is lower. It is worth noting that there were only two features which were chosen by both techniques. Tables 3 and 4 are resembling each other. There was a large overlap between Normal and Other classes in all the matrices. In fact, two largest sources of misclassifications are predicting instances of Normal class as Other (339, 341, and 418 respectively) and predicting instances of Other class as Normal (647, 640, and 893 respectively). Misclassifying Other as Normal was the main source of the performance degradation when using the subset from the statistical approach. The second largest overlap is between $\mathrm{AF}$ and Other classes. Finally, the least represented class (Noise) gets the lowest $F_{1}$ score per class. It is not sur- 

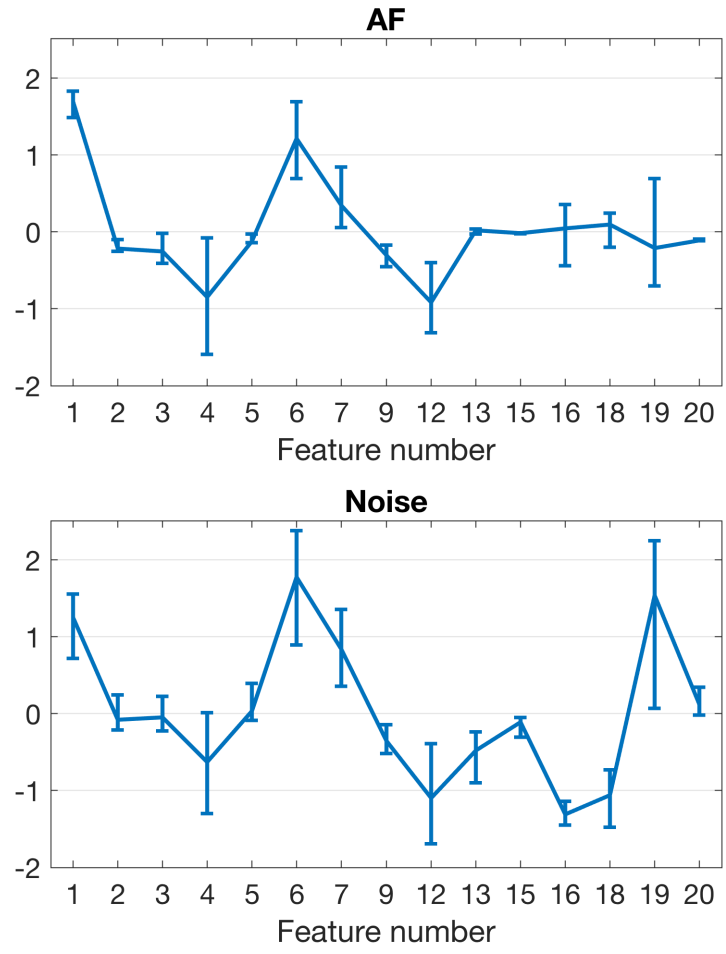

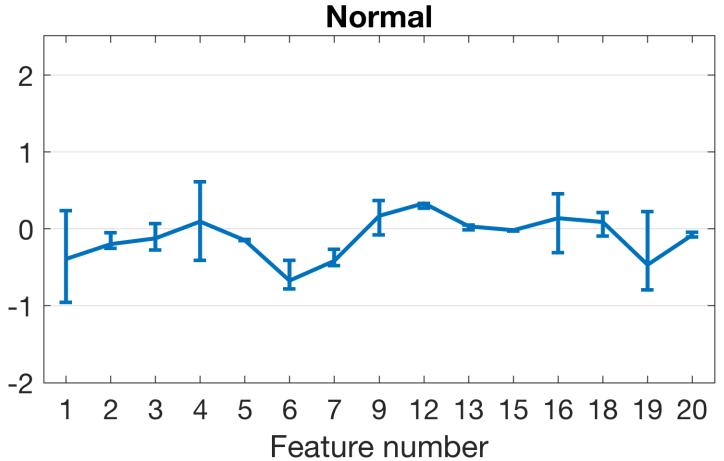

Other

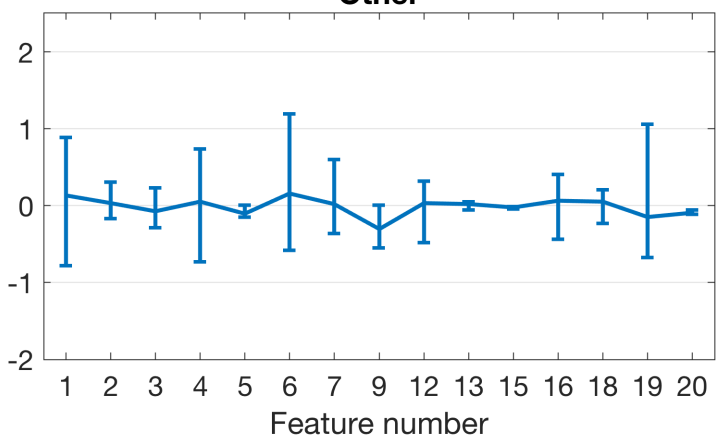

Figure 1. Plots illustrating distributions of 15 features selected by the RFE for each class in the dataset. Lines show medians. Bars depict and interquartile ranges between $25 \%$ and $75 \%$ percentiles. For visual purposes, the hyperbolic tangent function was applied to all values of the features. Next, each feature was scaled using z-score method. The plots depict statistics for the scaled features.

prising as the classifier is maximizing the overall accuracy, thus, it is more important to correctly classify as many as possible of the examples of the most representative class (i.e., Normal). The least representative class becomes the least important one from the point of view of the average accuracy. Note, however, that for the considered task the goal is to maximize the mean $F_{1}$ score, which is negatively affected by low individual $F_{1}$ scores. In all tables there are many instances of Noise class which were predicted either as Normal or Other. Therefore, for the future work it will be important to improve the correctness of predicting instances from Noise class.

\section{Conclusion}

The presented results allow concluding that it is possible to significantly decrease the complexity of the existing solutions to AF classification problem, both in terms of the extracted number of features and transforms applied to raw signals, without sacrificing the classification performance of the solution. This, in turn, increases the feasibility of deploying such solutions on resource-limited devices.

Acknowledgment: The research reported here was supported and funded by the PERCCOM Erasmus Mundus
Program of the European Union (PERCCOM-FPA 20130231). AA would like to express her gratitude to all the partner institutions, sponsors, and researchers involved in the program.

\section{References}

[1] Clifford GD, Liu C, Moody B, wei H. Lehman L, Silva I, Li Q, Johnson A, Mark RG. Af classification from a short single lead ecg recording: the physionet/computing in cardiology challenge 2017. Computing in Cardiology 2017;44:1.

[2] Andreotti F, Carr O, Pimentel MA, Mahdi A, De Vos M. Comparing feature-based classifiers and convolutional neural networks to detect arrhythmia from short segments of ecg. Computing in Cardiology 2017;44:1.

[3] Guyon I, Weston J, Barnhill S, Vapnik V. Gene selection for cancer classification using support vector machines. Machine learning 2002;46(1-3):389-422.

Address for correspondence:

Name: Urban Wiklund

Full postal address: SE-901 87 Sweden, Umeå, Umeå University, Department of Radiation Sciences, Biomedical Engineering. E-mail address: Urban.Wiklund@umu.se 